\title{
Correction to: High extracellular ATP levels released through pannexin-1 channels mediate inflammation and insulin resistance in skeletal muscle fibres of diet-induced obese mice
}

\author{
Gonzalo Jorquera $^{1,2}$ (D) $\cdot$ Roberto Meneses-Valdés $^{2}$ (D) Giovanni Rosales-Soto ${ }^{2,3} \cdot$ Denisse Valladares-Ide $^{4}$ (D)

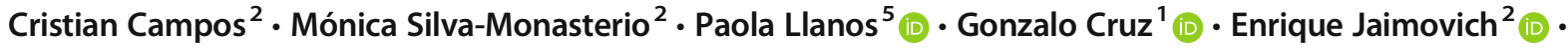 \\ Mariana Casas $^{2}$ (D)
}

Published online: 1 April 2021

(C) Springer-Verlag GmbH Germany, part of Springer Nature 2021

\section{Correction to: Diabetologia}

https://doi.org/10.1007/s00125-021-05418-2

The affiliations for Denisse Valladares-Ide and Paola Llanos have been corrected.

Publisher's note Springer Nature remains neutral with regard to jurisdictional claims in published maps and institutional affiliations.

The online version of the original article can be found at https://oi.org/ $10.1007 / \mathrm{s} 00125-021-05418-2$

\section{Gonzalo Jorquera}

gonzalo.jorquera@uv.cl

$\bowtie$ Mariana Casas

mcasas@med.uchile.cl

1 Centro de Neurobiología y Fisiopatología Integrativa (CENFI), Instituto de Fisiología, Facultad de Ciencias, Universidad de Valparaíso, Valparaíso, Chile

2 Centro de Estudios de Ejercicio, Metabolismo y Cáncer, Programa de Fisiología y Biofísica, Instituto de Ciencias Biomédicas, Facultad de Medicina, Universidad de Chile, Santiago, Chile

3 Facultad de Ciencias de la Educación, Universidad San Sebastián, sede Bellavista, Santiago, Chile

4 Instituto de Ciencias de la Salud, Universidad de O'Higgins, Rancagua, Chile

5 Instituto de Investigación en Ciencias Odontológicas, Facultad de Odontología, Universidad de Chile, Santiago, Chile 